\title{
A SHOOTING APPROACH TO THE LORENZ EQUATIONS
}

\author{
S. P. HASTINGS AND W. C. TROY
}

\begin{abstract}
We announce and outline a proof of the existence of a homoclinic orbit of the Lorenz equations. In addition, we develop a shooting technique and two key conditions, which lead to the existence of a one-to-one correspondence between a set of solutions and the set of all infinite sequences of 1's and 3's.
\end{abstract}

\section{INTRODUCTION}

The system of equations discovered by Lorenz [6] is found in computer simulations to have chaotic behavior, by practically any definition of that term. A survey appears in [7]. However, aside from local results and various kinds of bifurcation analysis, little has been proved about these equations.

We have now been able to prove the existence of a homoclinic orbit for an open set of parameter values. That is, there is a nonconstant solution tending to the same equilibrium point, in this case $(0,0,0)$, at $\pm \infty$. Such a solution has long been conjectured to exist and is recognized as an important feature of these equations. Further, we have proved a theorem, which reduces the question of whether there are "chaotic" orbits to one which, in principle, can be handled for an open set of parameter values with the techniques of rigorous numerical analysis, such as interval arithmetic [1]. In other words, the amount of computation required for rigorous verification of the hypotheses of our second theorem is finite; whether it is practical remains an open question at this time. Computer assisted proofs generally leave a gap in understanding, but with Theorems 1 and 2, we believe the gap is smaller than before.

The use of a "shooting" technique to obtain a homoclinic orbit is clearly suggested by numerical integrations. However, its analytic implementation is difficult and has not been done before, to our knowledge. The proof of the second theorem is easier. Its conclusion is interesting because it discusses the existence of chaos without reference to any bifurcation phenomena. The hypotheses appear to be true (based on standard numerical integrations) for the "classical" parameter values used for the Lorenz equations.

Our approach is more elementary than some other approaches to these equations, such as geometric models, which have given deep insights about chaotic behavior but have not been shown to apply to the system which motivated all this work. Instead of Poincare maps we use simple one parameter "shooting." Therefore, this is a further application of methods begun in [5] and continued in $[3,4]$. Techniques from [8] are also important.

Received by the editors January 7, 1992 and, in revised form, April 20, 1992.

1991 Mathematics Subject Classification. Primary 58F15, 58F13. 
Theorem 1. For each $(s, q)$ in some neighborhood of the point $(10,1)$ there is an $R$ in the interval $(1,1000)$ such that the system of equations

$$
\begin{aligned}
x^{\prime} & =s(y-x), \\
y^{\prime} & =R x-y-x z, \\
z^{\prime} & =x y-q z
\end{aligned}
$$

has a homoclinic orbit.

We chose $q=1$ as the point to do our analysis instead of the usual value of $8 / 3$, purely for convenience in the many numerical calculations. The lack of precision in the $R$ value could easily be reduced dramatically with computer assistance. Also, the range of parameters $(s, q)$ for which an $R$ exists could be expanded greatly; but it would be more desirable to find a method that would reveal the set of such $(s, q)$ analytically, by proving an extension of Lemma 2. Further homoclinic orbits for a given $(s, q)$ could, in principle, also be found by our method.

\section{OUTLINE OF PROOF THAT THERE IS A HOMOCLINIC ORBIT}

For any $R>1$, the equilibrium point $(0,0,0)$ is unstable and has a onedimensional unstable manifold, which we denote by $\gamma$. We analyze the behavior of this manifold for $R$ close to 1 and for $R=1000$. We prove two lemmas, which deal with a solution $p(t)=(x(t), y(t), z(t))$ on the "positive" branch $\gamma^{+}$of the unstable manifold, so that $p(t) \rightarrow(0,0,0)$ as $t \rightarrow-\infty$, and $x(t), y(t)$, and $z(t)$ are all positive for large negative $t$.

Lemma 1. For $(s, q)$ in some open neighborhood of $(10,1)$ and $R-1$ positive but sufficiently small, $x(t), y(t)$, and $z(t)$ are all positive on the entire line $-\infty<t<\infty$.

Lemma 2. For $(s, q)$ in some open neighborhood of $(10,1)$ and $R=1000$, $x(t)$ has at least one zero, $t_{1}$, and $x^{\prime}$ changes sign exactly once in $\left(-\infty, t_{1}\right]$.

Lemma 1 is easy to prove analytically, but Lemma 2 is much more difficult. With these two results, the existence of a homoclinic orbit is seen fairly quickly. We let $R^{*}$ be the infimum of all values of $R>1$ for which the behavior in Lemma 2 occurs. For $R=R^{*}$ and $(x(t), y(t), z(t))$ on $\gamma^{+}$, we can show that either (a) $x$ and $x^{\prime}$ vanish simultaneously, or (b) after $\tau_{1}$, the first zero of $x^{\prime}, x$ decreases as long as the solution exists but never becomes negative. This requires eliminating other options, such as the bifurcation from some finite point of new zeros of $x^{\prime}$ at $R^{*}$.

In case (a), $x=y=0$ at some finite time $t$, and then the uniqueness theory for initial value problems implies that $x$ and $y$ are identically zero. This is impossible on $\gamma^{+}$. In case (b), it is clear that $(x, y, z)$ must approach an equilibrium point as $t \rightarrow \infty$. Only a little more effort is needed to show that this is the origin and the orbit is homoclinic.

The proof of Lemma 2 requires a careful study of $\gamma^{+}$. The value $R=1000$ is, of course, rather arbitrary. It is not hard to prove that on some initial interval $\left(-\infty, t_{0}\right], x, y$, and $z$ increase monotonically, reaching a point 
where $y=1,0.096 \leq x \leq 0.1$, and $x^{2} / 20<z<0.1$. From this point the result would follow easily with computer assistance; but in our opinion, ingenuity, and considerable effort, is required to follow the solution analytically. We show that $x, y$, and $z$ continue to rise at least until they reach the levels $z=1000,126.4<x<135.6$, and $798<y<1000$. From this region $y$, and eventually $x$ and $z$, begin to fall. It is apparent that at a point where $y$ becomes negative, $z$ must be greater than $\mathrm{R}$. In fact, we show that $y=0$ at a point where $155<x<189$ and $z>10.4 x$.

To obtain these inequalities we use (1) for initial estimates over suitable intervals, and then iterate to obtain better bounds. Use is made of the functions $S=\frac{1}{2}\left(y^{2}+z^{2}\right)-50 x^{2}$ and $Q=z-x^{2} / 20$. A continuation of this process into the region where $y$ is negative requires some "tricks" but finally yields the result. The details have been submitted elsewhere.

\section{CRITERIA FOR THE EXISTENCE OF COMPlicAted SOlutions}

In this section we give a theorem with the conclusion that for some values of $(s, q, R)$, equation (1) has solutions with very complicated behavior, in a sense to be made precise. The hypotheses of this theorem seem difficult to check analytically; however, the result seems to us to be an improvement over previous work because these hypotheses can be confirmed by examining only a compact segment of $\gamma^{+}$, together with a set of solutions $p$ such that $p(0)$ is in a compact line segment. This line segment lies in the intersection of the two planes $x=y$ and $z=R-1$. The solutions only have to be followed over compact time intervals, suggesting that the hypotheses can be checked rigorously with computer assistance.

Standard numerical analysis indicates that the hypotheses are satisfied, for some parameters, in a robust fashion so that the errors in the numerical analysis should not be so great that the result is false. This reinforces our hope that the theorem can be shown to apply to (1).

We have two principle hypotheses for Theorem 2. The first is an extension of Lemma 2.

Condition A. If $p$ is a solution of (1) with $p(0) \in \gamma^{+}$, then $x^{\prime}$ has at least five sign changes and $x$ has at least one zero. If $\tau_{1}<\tau_{2}<\tau_{3}<\tau_{4}<\tau_{5}$ are the first five sign changes of $x^{\prime}$, while $t_{1}<t_{2}$ are the first two zeros of $x$, then $\tau_{1}<t_{1}<\tau_{2}<\tau_{3}<\tau_{4}<\tau_{5}<t_{2}$. (If $x$ does not have a second zero, set $t_{2}=\infty$.)

This condition is obviously more restrictive than the conclusion of Lemma 2. When $(s, q)=(10,1)$, standard numerical integrations suggest that it holds for $R$ approximately in the range $(8.2,17.2)$. If $q=8 / 3$, then the $R$ range becomes about $(14,46.6)$. Unpredictable behavior exists outside of this range, and a straight forward extension of our theorem would partly explain this, but be harder to check rigorously.

Before stating our second hypothesis, we must describe the "shooting" procedure used to obtain the complicated solutions. The method is to choose initial conditions $p(0)$ in a certain line segment in the plane $x=y$ and give an inductive procedure for varying $p(0)$ to obtain more and more complex behavior.

To specify this line segment, suppose that Condition $A$ is satisfied. Then the 
branch $\gamma^{+}$of $\gamma$ first crosses the plane $x=y$ at some point $p_{1}$, which can be shown to lie in the region $z>R-1$. Also, since $R>1$, there is an equilibrium point $p_{0}$ of (1) in this plane in the positive octant. At $p_{0}, z=R-1$. Our shooting set is the line segment $L$ connecting $p_{0}$ and $p_{1}$.

The idea of our second condition is, roughly, that solutions starting on $L$ do not gain or lose sign changes of $x^{\prime}$ by bifurcation as the initial point changes on $L$. Stated that way, however, it appears necessary to follow these solutions on $0 \leq t<\infty$, clearly not possible numerically. Instead, we consider solutions starting along the line where zeros of $x^{\prime}$ bifurcate and follow these backwards. This is the line $\mathrm{M}$ defined by the equations $x=y, z=R-1$. Note that this line intersects L only at $p_{0}$. We will explain below why this should require only a finite amount of computation.

Condition B. Suppose $p$ is a nonconstant solution of (1) such that $p(0) \in M$. Then at least one of the following is true.

(2a) $p(t) \notin L$ for $t<0$

(2b) In some interval containing $t=0, x \neq 0$ and $x^{\prime}$ changes sign four times.

To check Condition A numerically, it is first necessary to give estimates that show that $\gamma^{+}$intersects a specific planar rectangle close to, but not including, the origin. It must then be shown that every solution starting in this rectangle behaves as described in the condition. This process is difficult, because current methods of interval arithmetic lose about 10 decimal places of accuracy for every time unit of integration for this system; however it has been successfully carried out by Hassard and Zhang [2].

To check Condition B, we suggest the use of the well-known result [7] that the ellipsoid $E$ defined by the inequality

$$
x^{2}+\frac{10}{R} y^{2}+\frac{10}{R}(z-2 R)^{2} \leq 40 R
$$

is a positively invariant set for (1) for a range of values of $q$ and $s$. The line segment $L$ lies in $E$. We consider initial points $p(0)$ on the (different) line segment $M \cap E$. Assuming that $p$ is not constant and (2b) cannot be verified, we would integrate from $p(0)$ backwards in $t$ and show that the solution leaves $E$ before intersecting the line segment $L$. Once $p$ leaves $E$ as $t$ decreases, it cannot reenter $E$ at a lower $t$ value. By its nature, a single integration using interval arithmetic can verify Condition B for an interval of initial conditions on $M$ around $p(0)$. The practical difficulty, which up to now has prevented us from completing this step, is that the length of these intervals is quite small, so that several thousand initial conditions must be considered. A local analysis around $p_{0}$ results in a bound on the length of the time intervals in our integrations.

We can now state our second theorem.

Theorem 2. Suppose that Conditions A and B hold for some $(s, q, R)$. Suppose also that two of the eigenvalues of the linearized system around $p_{0}$ are complex. Moreover, suppose that $\left\{M_{j}\right\}$ is any infinite sequence of 1 's and 3 's. Then there is a solution $p=(x, y, z)$ of (1) such that $x$ has an infinite number of zeros in $0<t<\infty$, and if $\left\{t_{i}\right\}$ is the sequence of consecutive zeros of $x$ in 
$[0, \infty)$ and $\sigma_{i}$ is the number of sign changes of $x^{\prime}$ in $\left(t_{i}, t_{i+1}\right)$, then $\sigma_{i}=M_{i}$ for $1 \leq i<\infty$.

Outline of Proof. Parametrize $L$ by setting $p_{\alpha}(0)=\alpha p_{0}+(1-\alpha) p_{1}$, for $0 \leq \alpha \leq 1$. The proof proceeds by induction, choosing a sequence of $\alpha$ 's giving more and more of the prescribed numbers of critical points between zeros of $x$.

Because two of the eigenvalues of the linearized system around $p_{0}$ are complex, it follows that if $\alpha$ is close to 1 , then $p$ crosses the plane $y=x$ in $0<t<\infty$ before any possible zero of $x$. On the other hand, for small $\alpha$, $x$ decreases monotonically to below 0 , after which $x^{\prime}$ changes sign at least four times before $x=0$ a second time. Therefore, the first positive zero of $x$, $t_{1}(\alpha)$, is defined and continuous on some maximal interval of the form $[0, \bar{\alpha})$, where $\bar{\alpha}<1$.

If $x_{\alpha}$ has at least $\mathrm{n}$ positive zeros $t_{1}(\alpha), \ldots, t_{n}(\alpha)$, let $t_{n+1}(\alpha)$ denote the $(n+1)$ st positive zero of $x$ if this exists, or else $t_{n+1}(\alpha)=\infty$. Also, for $1 \leq i \leq n$, let $\sigma_{j}=\sigma_{j}(\alpha)$ denote the number of sign changes of $x^{\prime}$ in $\left[t_{i}, t_{i+1}\right)$.

Suppose that $t_{n}()$ is continuous on some interval $I_{n} \subset[0, \bar{\alpha})$. We define three subsets of $I_{n}$ as follows, where the dependence of the $t_{j}$ and $\sigma_{j}$ on $\alpha$ is again understood:

$$
\begin{aligned}
& A_{n}\left(I_{n}\right)=\left\{\alpha \in I_{n} \mid t_{n+1}<\infty, \sigma_{n}=1 \text { and } \sigma_{n+1} \geq 4\right\}, \\
& B_{n}\left(I_{n}\right)=\left\{\alpha \in I_{n} \mid t_{n+1}<\infty, \sigma_{n}=3 \text { and } \sigma_{n+1} \geq 4\right\}, \\
& C_{n}\left(I_{n}\right)=\left\{\alpha \in I_{n} \mid t_{n+1}<\infty, \sigma_{n} \geq 4 \text { and } \sigma_{n+1} \geq 4\right\} .
\end{aligned}
$$

We prove the following, which imply the theorem.

(i) Let $I_{1}=(0, \bar{\alpha})$. Then $A_{1}\left(I_{1}\right), B_{1}\left(I_{1}\right)$, and $C_{1}\left(I_{1}\right)$ are all nonempty.

(ii) If, for some $\mathrm{n}$ and some $I_{n}, A_{n}=A_{n}\left(I_{n}\right), B_{n}$, and $C_{n}$ are all nonempty, then there are intervals $I_{n+1} \subset I_{n}$ and $I_{n+1}^{\prime} \subset I_{n}$ such that

(a) $t_{n+1}()$ is continuous on $I_{n+1}$ and on $I_{n+1}^{\prime}$;

(b) $I_{n+1} \cap A_{n}$ and $I_{n+1}^{\prime} \cap B_{n}$ are nonempty;

(c) The sets $A_{n+1}\left(I_{n+1}\right), B_{n+1}\left(I_{n+1}\right), C_{n+1}\left(I_{n+1}\right), A_{n+1}\left(I_{n+1}^{\prime}\right), B_{n+1}\left(I_{n+1}^{\prime}\right)$, and $C_{n+1}\left(I_{n+1}^{\prime}\right)$ are all non-empty.

We do not have space for the details here, and they have been submitted elsewhere. Condition B is used to show that as $\alpha$ varies, the number of sign changes of $x_{\alpha}^{\prime}$ between consecutive zeros of $x$ can decrease from four or more to three, or from two or three to one, only when the fourth or second of these sign changes tends to infinity on the $t$ axis. The number of sign changes of $x^{\prime}$ cannot jump directly from four or more to one without passing through an open set of $\alpha$ 's where there are three.

These theorems have a few simple corollaries, which we will mention when details of the proofs are published. Since the divergence of the Lorenz vector field is negative, volumes are reduced by the flow. We hope to investigate whether our results have any consequences about the existence of "strange attractors." 


\section{REFERENCES}

1. O. Aberth, Precise numerical analysis, William C. Brown Publishers, Dubuque, IA, 1988.

2. B. Hassard and J. Zhang (to appear).

3. S. Hastings and J. B. McLeod, On the periodic solutions of a forced second-order equation, Nonlinear Science, 1 (1991), 225-245.

4. S. Hastings and J. B. McLeod, On the chaotic motion of a forced pendulum, Amer. Math. Monthly (to appear).

5. S. Hastings and W. Troy, Oscillating solutions of the Falkner-Skan equation for positive $\beta$, J. Differential Equations 71 (1988), 123-144.

6. E. N. Lorenz, Deterministic non-periodic flow, J. Atmospheric Sci. 20 (1963), 130-141.

7. C. Sparrow, The Lorenz equations: bifurcations, chaos, and strange attractors, Applied Math. Sci. vol. 41, Springer-Verlag, Berlin and New York, 1982.

8. W. Troy, The existence of bounded solutions of the Kuramoto-Sivashinskii equations, J. Differential Equations 82 (1989), 269-313.

Department of Mathematics, University of Pittsburgh, Pittsburgh, Pennsylvania 15260

E-mail address: SPH@MTHSN4.Math.P.H.edu

E-mail address: Troy@VMS.CIS.PITT.EDU 